Revue internationale P.M.E.

Économie et gestion de la petite et moyenne entreprise

\title{
Les facteurs de succès des entreprises à croissance rapide en Allemagne
}

\section{Michael Woywode et Vera Lessat}

Volume 14, numéro 3-4, 2001

URI : https://id.erudit.org/iderudit/1008696ar

DOI : https://doi.org/10.7202/1008696ar

Aller au sommaire du numéro

\section{Éditeur(s)}

Presses de l’Université du Québec

ISSN

0776-5436 (imprimé)

1918-9699 (numérique)

Découvrir la revue

\section{Citer cet article}

Woywode, M. \& Lessat, V. (2001). Les facteurs de succès des entreprises à croissance rapide en Allemagne. Revue internationale P.M.E., 14(3-4), 17-43. https://doi.org/10.7202/1008696ar

\section{Résumé de l'article}

Cette étude analyse les entreprises d'Allemagne de l'Ouest connaissant une croissance de l'emploi particulièrement importante. Nous désignons comme entreprises à croissance rapide celles qui appartiennent aux $10 \%$ des entreprises ayant la plus forte croissance de l'emploi. Après une discussion théorique sur les déterminants de la croissance d'entreprise, nous développerons une série d'hypothèses dont nous vérifierons l'exactitude de façon empirique à l'aide de deux échantillons d'entreprises. Le premier échantillon comprend environ 11000 entreprises de l'industrie de transformation (secteur secondaire), de l'industrie du bâtiment, du commerce et de l'industrie des services en général (secteur tertiaire). Les données dont nous disposons proviennent des informations de la plus grande agence allemande de renseignements sur les crédits, CREDITREFORM. Le deuxième échantillon comporte des informations sur environ 4000 entreprises ouest-allemandes de l'industrie de transformation et de l'industrie de services liés à la production. Pour cet échantillon, les informations proviennent d'un sondage écrit. Nos résultats peuvent être résumés ainsi : nous confirmons les résultats d'enquêtes précédentes, qui attestent qu'un petit groupe d'entreprises à croissance rapide apporte une contribution essentielle à la croissance de l'emploi. En moyenne, les petites et les jeunes entreprises appartiennent plus que les grandes entreprises ou celles plus anciennes aux groupes d'entreprises à croissance rapide. En outre, nous découvrons que le secteur d'activité et la forme juridique choisie par l'entreprise influent sur la probabilité pour l'entreprise de faire partie des entreprises à croissance rapide. Les stratégies des entreprises en matière de recherche et développement ou d'exportation, mais aussi les caractéristiques personnelles de l'entrepreneur telles que son âge et son diplôme sont des déterminants majeurs de la forte croissance d'une entreprise.
Ce document est protégé par la loi sur le droit d'auteur. L'utilisation des services d’Érudit (y compris la reproduction) est assujettie à sa politique d'utilisation que vous pouvez consulter en ligne.

https://apropos.erudit.org/fr/usagers/politique-dutilisation/ 


\title{
Les facteurs de succès des entreprises à croissance rapide en Allemagne
}

\author{
Michael WOYWODE
}

Université de Mannheim

Vera LESSAT

DZ Bank de Francfort

\section{MOTS CLÉS}

\section{Facteurs de succès - Croissance rapide - Allemagne de l'Ouest Secteur d'activité - Stratégies - Entrepreneur}

\begin{abstract}
RÉSUMÉ
Cette étude analyse les entreprises d'Allemagne de l'Ouest connaissant une croissance de l'emploi particulièrement importante. Nous désignons comme entreprises à croissance rapide celles qui appartiennent aux $10 \%$ des entreprises ayant la plus forte croissance de l'emploi. Après une discussion théorique sur les déterminants de la croissance d'entreprise, nous développerons une série d'hypothèses dont nous vérifierons l'exactitude de façon empirique à l'aide de deux échantillons d'entreprises. Le premier échantillon comprend environ 11000 entreprises de l'industrie de transformation (secteur secondaire), de l'industrie du bâtiment, du
\end{abstract}

\section{LES AUTEURS}

MICHAEL WOYWODE enseigne actuellement la gestion des affaires à l'Université de Mannheim. Il a étudié à Saint-Gall, Paris et Stanford et a reçu un doctorat en Sciences économiques de l'Université de Mannheim en 1997. II est aussi responsable de l'Institut pour les études de gestion (IBU) à l'Université de Karlsruhe. Ses recherches se concentrent sur l'explication de la croissance des entreprises et la dynamique industrielle de même que sur l'étude des processus de changement d'organisation interne. Courriel : <mwoywode@yahoo.com>.

VERA LESSAT travaille comme analyste industrielle à la DZ Bank de Francfort, en Allemagne. Elle a étudié les sciences économiques à Dortmund et à Augsbourg, en Allemagne, et a terminé sa thèse de doctorat en 1994. Ensuite, elle a travaillé en tant que chercheuse à l'Institut allemand pour la recherche, à Berlin et au Centre européen pour la recherche (ZEW), à Mannheim. Récemment, elle a terminé une recherche sur les compagnies de pointe en Allemagne de l'Est. Elle travaille également pour l'industrie pharmaceutique et biotechnologique. 
commerce et de l'industrie des services en général (secteur tertiaire). Les données dont nous disposons proviennent des informations de la plus grande agence allemande de renseignements sur les crédits, CREDITREFORM. Le deuxième échantillon comporte des informations sur environ 4000 entreprises ouest-allemandes de l'industrie de transformation et de l'industrie de services liés à la production. Pour cet échantillon, les informations proviennent d'un sondage écrit. Nos résultats peuvent être résumés ainsi : nous confirmons les résultats d'enquêtes précédentes, qui attestent qu'un petit groupe d'entreprises à croissance rapide apporte une contribution essentielle à la croissance de l'emploi. En moyenne, les petites et les jeunes entreprises appartiennent plus que les grandes entreprises ou celles plus anciennes aux groupes d'entreprises à croissance rapide. En outre, nous découvrons que le secteur d'activité et la forme juridique choisie par l'entreprise influent sur la probabilité pour l'entreprise de faire partie des entreprises à croissance rapide. Les stratégies des entreprises en matière de recherche et développement ou d'exportation, mais aussi les caractéristiques personnelles de l'entrepreneur telles que son âge et son diplôme sont des déterminants majeurs de la forte croissance d'une entreprise.

\begin{abstract}
This paper discusses the determinants of high-growth firms in Germany. High-growth firms are defined as the «top ten percent companies» with respect to employment growth and turnover growth. We review the relevant literature concerning firm growth, develop a number of hypotheses, and test these predictions by considering the growth rates of enterprises in two separate samples containing West German firms. The first sample contains approximately 11000 companies from manufacturing, construction, trade, finance and services. This data set is based on firm level information of a credit rating agency. The firms were tracked over a six-year period. The second sample contains information on 4000 West German firms from manufacturing and some business services. In this case, firm level information was gathered by interviewers and covers a four-year interval. Our results can be summarised as follows. We find that smaller firms have a higher likelihood of being among the "top ten percent companies». Furthermore, we find evidence that the industry affiliation and the legal form adopted by companies also affect the likelihood of firms to belong to the "top ten percent companies». Firm strategies regarding $R-D$ and exports as well as personal characteristics of the entrepreneur like the age and the educational degree are important for explaining high-growth performance, too.
\end{abstract}

\title{
RESUMEN
}

En este estudio analizamos empresas del oeste de Alemania cuyo crecimiento es particularmente elevado. Empresas de crecimiento rápido son, desde nuestro punto de vista, aquellas que pertenecen al $10 \%$ de empresas con mayor expansión. Discutimos la bibliografia relevante en cuanto a los determinantes del crecimiento empresarial y desarrollamos un número de hipótesis que serán verificados empíricamente a través de muestreos aplicados a dos empresas.

El primer muestreo incluye aproximadamente 11.000 empresas de la industria de manufación, empresas del ramo de la construción, el comercio u otras empresas del sector servicios. Los datos provienen del archivo alemán más amplio para informaciones de crédito, el CREDITREFORM.

Revue internationale P.M.E., vol. 14, $\mathrm{n}^{\text {os }} 3-4,2001$

(C) 2002 - Presses de l'Université du Québec

Édifice Le Delta I, 2875, boul. Laurier, bureau 450, Sainte-Foy, Québec G1V 2M2 • Tél. : (418) 657-4399 - www.puq.uquebec.ca

Tiré de : Revue internationale P.M.E., vol. 14, nos $3-4$, sous la direction de Pierre-André Julien. 
El segundo muestreo incluye informaciones sobre aproximadamente 4000 empresas occidentales-alemanes de la industria de manufación y del sector servicios que tienen que ver con la producción. En este último caso, las informaciones de las empresas provienen de un cuestionario aplicado en las mismas empresas.

Nuestros resultados se resumen de la siguiente manera: confirmamos las conclusiones de investigaciones anteriores aplicadas a un pequeño grupo de empresas de crecimiento rápido que contribuye esencialmente a la expansión de la empresa. Se muestra que es más probable que empresas pequeñas o nuevas pertenezcan al grupo de las empresas con crecimiento rápido que empresas grandes y consolidadas. Además descubrimos que la pertenecia a un ramo y la forma jurídica elegida por la empresa también ejercen una influencia sobre la probabilidad de que una empresa pueda incluirse en el grupo de las empresas de crecimiento rápido.

Estrategias de empresas como la estrategia de investigación, de desarrollo y de exportación así como las características individuales de un empresario, es decir su edad y su formación por ejemplo, son indicadores del rendimiento relativo al crecimiento de una empresa.

\section{ZUSAMMENFASSUNG}

In dieser Studie analysieren wir westdeutsche Unternehmen mit einem besonders hohen Beschäftigungswachstum. Als schnell wachsende Unternehmen bezeichnen wir dabei diejenigen Unternehmen, die zu den zehn Prozent mit dem höchsten Beschäftigungswachstum gehören. Wir diskutieren die relevante Literatur bezüglich der Determinanten des Unternehmenswachstums und entwickeln eine Anzahl von Hypothesen, die anhand von zwei Unternehmensstichproben empirisch überprüft werden. Die erste Stichprobe beinhaltet etwa 11.000 Unternehmen des Verarbeitenden Gewerbes, des Baugewerbes, des Handels und des sonstigen Dienstleistungssektors. Dieser Datensatz basiert auf Informationen der größten deutschen Kreditauskunftei CREDITREFORM. Die zweite Stichprobe beinhaltet Informationen über etwa 4000 westdeutsche Unternehmen aus dem Verarbeitenden Gewerbe und dem produktionsnahen Dienstleistungsbereich. In diesem Fall stammen die Unternehmensinformationen aus einer schriftlichen Unternehmensbefragung. Unsere Resultate können folgendermaßen zusammengefaßt werden: Wir bestätigen den Befund früherer Untersuchungen, daß eine kleine Gruppe von schnell wachsenden Unternehmen einen ganz wesentlichen Beitrag zum Beschäftigungswachstum leistet. Es zeigt sich, daß kleinere und jüngere Unternehmen eine höhere Wahrscheinlichkeit haben, zur Gruppe der schnell wachsenden Unternehmen zu gehören als große, alte Unternehmen. Weiterhin finden wir heraus, daß die Branchenzugehörigkeit und die Rechtsform, die von den Unternehmen gewählt wurden, ebenfalls die Wahrscheinlichkeit beeinflussen, daß ein Unternehmen zur Gruppe der schnell wachsenden Unternehmen gehört. Unternehmensstrategien, wie die Forschungs- und Entwicklungsstrategie oder die Exportstrategie aber auch persönliche Charakteristika des Unternehmers, wie sein Alter und sein Bildungsabschluß sind ebenfalls wichtige Prädiktoren der Wachstumsperformance eines Unternehmens.

Revue internationale P.M.E., vol. 14, $\mathrm{n}^{\text {os }} 3-4,2001$

(C) 2002 - Presses de l'Université du Québec

Édifice Le Delta I, 2875, boul. Laurier, bureau 450, Sainte-Foy, Québec G1V 2M2 • Tél. : (418) 657-4399 - www.puq.uquebec.ca

Tiré de : Revue internationale P.M.E., vol. 14, nos 3-4, sous la direction de Pierre-André Julien. 


\section{Introduction}

Le grand intérêt porté depuis quelques décennies à la création d'entreprises est lié avant tout au besoin de créer de nombreux emplois. C'est en particulier Birch (1981, 1987) qui a attiré l'attention depuis la fin des années 1970 aux États-Unis sur l'importance économique des petites et nouvelles entreprises concernant la croissance de l'emploi. Les travaux théoriques fondamentaux à ce sujet ont leur origine dans les études de Gibrat (1931) et de Simon et Bonini (1956), dans lesquelles les liens entre taille, croissance des entreprises et structure de marché sont examinés. Encouragées par les travaux de Birch, beaucoup d'études empiriques ont été menées sur le développement des petites et jeunes entreprises et leur contribution à l'emploi. Les articles récents importants à ce propos relèvent, par exemple, des travaux de Brock et Evans (1986), Evans (1987 a et b), Hall (1987), Dunne, Roberts et Samuelson (1989 a et b), Sutton (1997) ou, quant à l'économie allemande, ceux de Brüderl, Preisendörfer et Ziegler (1996), Wagner (1994) ainsi que Harhoff, Stahl et Woywode (1998).

Un résultat important et jusque-là peu considéré est le fait qu'à long terme seule une petite partie des entreprises apporte une contribution notable à l'emploi. Un grand nombre de jeunes et petites entreprises disparaissent du marché, entraînant des licenciements. De plus, on découvre que, pour un nombre relativement important d'entreprises, le nombre d'employés reste quasi constant pendant une longue période et que seule une faible part des entreprises réalise une forte croissance de l'emploi (Brüderl et al., 1996). Storey (1995), dans une étude sur la Grande-Bretagne, révèle que $10 \%$ d'une cohorte d'entreprises en démarrage sont à elles seules responsables de $70 \%$ de la croissance de l'emploi sur une période de sept ans. Dans une étude sur l'Allemagne, Brüderl et Preisendörfer (2000) montrent que seulement $4 \%$ des nouvelles entreprises connaissent une forte croissance des effectifs sur une période de cinq ans ; mais ces $4 \%$ d'entreprises sont responsables d'un tiers des emplois créés sur une période de cinq ans. Si ces résultats étaient confirmés par d'autres études menées sur des échantillons plus larges, la politique économique devrait éventuellement reconsidérer sa politique d'aide aux créations d'entreprises. Une aide plus ciblée sur les entreprises à haut potentiel de croissance aurait sans doute un impact plus fort en matière d'emploi. Pour cibler l'aide sur les entreprises à forte croissance, il est important de connaître les facteurs qui favorisent la croissance des entreprises. Dans cette perspective, notre enquête sur les facteurs à l'origine de la forte croissance de certaines entreprises contribue à fournir des éléments d'information essentiels pour définir une politique économique fructueuse.

Notre étude vise à identifier, de façon empirique à partir de larges échantillons de données sur les entreprises, les facteurs influant sur la croissance de l'emploi à l'échelle des entreprises. Dans cette étude, nous entendons par entreprises à forte croissance celles qui font partie des $10 \%$ qui connaissent la croissance la plus

Revue internationale P.M.E., vol. 14, $\mathrm{n}^{\text {os }} 3-4,2001$ 
importante. Les analyses empiriques sont fondées sur deux bases de données qui contiennent des informations sur des entreprises de l'Allemagne de l'Ouest. La première base de données s'appelle le Mannheimer Unternehmenspanel (MUP). Le MUP comprend environ 11000 entreprises dans tous les secteurs de l'économie, ces entreprises étant observées sur une période de six ans. Les données des entreprises sont mises à disposition par l'agence de renseignements CREDITREFORM. La deuxième base de données s'appelle le Mannheimer Innovationspanel (MIP). Le MIP contient des informations sur environ 4000 entreprises ouest-allemandes dans l'industrie de transformation du secteur secondaire et dans le secteur des services liés à la production. Les informations sur les entreprises du MIP proviennent d'enquêtes écrites et couvrent une période de quatre ans.

Nos résultats se résument ainsi : les petites et jeunes entreprises ont une plus forte probabilité d'appartenir au groupe des entreprises à forte croissance. En outre, nous observons que la branche à laquelle l'entreprise appartient influence aussi la probabilité d'appartenir au groupe des entreprises à forte croissance. En moyenne, les entreprises dans le secteur des services ont une plus forte chance d'appartenir à ce groupe que celles des autres secteurs. De plus, nous montrons que les entreprises à responsabilité limitée ont, elles aussi, de fortes chances de faire partie des entreprises à forte croissance. Nos résultats laissent croire que des investissements importants en recherche et développement ont des répercussions positives sur la croissance de l'entreprise. Les caractéristiques de l'entrepreneur sont également des facteurs essentiels quant à la croissance de l'entreprise. En effet, les entreprises ayant des dirigeants jeunes ayant fait des études supérieures appartiennent avec une assez forte probabilité à ce type d'entreprises.

Dans la section suivante, nous nous livrons tout d'abord à quelques réflexions théoriques fondamentales à propos de la croissance des entreprises et nous les étayons par une documentation s'y rapportant. Nous exposons un certain nombre d'hypothèses pour relier les différentes caractéristiques relatives au secteur d'activité, à l'entreprise et au dirigeant des entreprises à croissance rapide. La deuxième section est consacrée à la description des données utilisées. Dans la troisième section, nous présentons une analyse descriptive de la croissance de l'emploi dans les entreprises de nos échantillons ainsi qu'un exposé des résultats économétriques se rapportant aux déterminants de la croissance de l'entreprise.

Notre article se termine par une conclusion dans laquelle on ouvrira des perspectives pour de futures recherches.

\section{Aspects théoriques de la croissance des entreprises}

Dans un grand nombre d'études, la croissance est traitée de façon générale. En revanche, il existe très peu d'études se concentrant sur les entreprises à forte croissance. Pour cette raison, nous considérons notre étude comme de nature prospective,

Revue internationale P.M.E., vol. 14, $\mathrm{n}^{\text {os }}$ 3-4, 2001 
c'est-à-dire que nous ne développons pas de modèle d'explication à proprement parler, qui comprendrait tous les facteurs qui influent sur la probabilité pour une entreprise d'appartenir à ce groupe de gazelles. Notre objectif est plutôt d'étudier toute une série de facteurs qui, dans des études précédentes, se sont révélés essentiels pour la croissance des entreprises. La documentation, dans le domaine de l'économie industrielle et de la gestion d'entreprises, a identifié un grand nombre de facteurs pouvant aider à comprendre la croissance des entreprises. Ces facteurs peuvent se répartir en trois catégories : 1) les facteurs reliés au secteur d'activité et, par conséquent, au développement de la demande, à l'intensité de la technologie, à la taille minimale optimale de l'entreprise et à l'importance des barrières à l'entrée et à la sortie sur le marché, 2) les facteurs reliés à l'organisation comme la taille, l'âge de l'entreprise, sa stratégie ainsi que le statut juridique et la composition du capital et, enfin, 3) les facteurs reliés à la personnalité de l'entrepreneur, à son âge, à sa motivation, à sa formation et à son expérience.

1. Dans le cas du premier type de facteurs touchant explicitement le secteur d'activité, une des rares études est celle d'Audretsch (1995). Cet auteur analyse les relations entre la taille minimale optimale, l'intensité de la technologie et la croissance de l'entreprise. Il établit une corrélation entre cette taille dans une industrie et le taux de croissance des effectifs dans les entreprises créées récemment. Audretsch explique ces résultats de la manière suivante : les jeunes entreprises sont obligées d'atteindre rapidement leur taille minimale optimale sous peine de disparaître du marché, étant donné leurs désavantages par rapport à la concurrence. À propos de l'intensité des technologies d'une industrie, Audretsch démontre que le taux de croissance des entreprises du secteur de la haute technologie est supérieur à la moyenne.

Dunne, Roberts et Samuelson (1989 a et b) analysent les déterminants de la croissance des entreprises et l'échec de certaines entreprises américaines dans l'industrie de transformation. Ils montrent que les taux de croissance des entreprises varient sensiblement selon les différentes branches de l'industrie. Harhoff, Stahl et Woywode (1998) confirment aussi ces différences selon la spécificité des branches. Ils utilisent un échantillon d'environ 10000 entreprises ouest-allemandes, couvrant tous les secteurs de l'économie. Dans cette étude, on découvre que les entreprises du secteur des services sont caractérisées par des taux de croissance de l'emploi supérieurs à la moyenne. Almus et Nerlinger (1999) et Brüderl, Preisendörfer et Ziegler (1996) confirment également des différences significatives des taux de croissance de l'emploi selon la spécificité des branches. Johnson, Baldwin et Hinchley (1997) trouvent une relation étroite entre la dynamique du développement à l'intérieur d'une branche et le taux de croissance d'une entreprise. Plus exactement, ils apportent les arguments suivants : les taux de croissance des entreprises dans des branches en fort développement devraient être plus élevés que ceux des entreprises 
dans des branches en stagnation, voire en diminution. Les jeunes marchés en expansion sont en général caractérisés par des barrières à l'entrée sur le marché relativement basses et, par conséquent, par une forte entrée ou sortie sur le marché. Des occasions de croissance plus ou moins grandes pour les entreprises découlent du niveau de développement de leur secteur d'activité.

2. Dans la majeure partie des ouvrages portant sur la croissance des entreprises, on analyse les relations entre les caractéristiques des entreprises et leur croissance. Les travaux d'Evans (1987 a et b), Dunne, Roberts et Samuelson (1989 a et b), Harhoff, Stahl et Woywode (1998) ainsi que Almus et Nerlinger (2000) montrent que les taux de croissance des effectifs diminuent avec la taille des entreprises. En outre, la plupart de ces études confirment une relation négative entre l'âge des entreprises et leur taux de croissance en effectifs. Les entreprises ne se différencient pas uniquement par leur taille et leur âge, mais aussi par leur statut juridique et la composition de leur capital. En général, on peut distinguer les entreprises dépendantes et les entreprises indépendantes. Une entreprise est considérée comme dépendante lorsque la majorité de son capital est aux mains d'autres entreprises. Être dépendant peut être un avantage pour l'entreprise concernée. Comparées aux entreprises indépendantes, les dépendantes possèdent souvent un meilleur accès aux ressources sur des marchés par nature imparfaits, ce qui est primordial pour prospérer sur ces marchés. Ainsi, les entreprises dépendantes devraient enregistrer des taux de croissance plus élevés que les entreprises indépendantes.

Dunne, Roberts et Samuelson (1989 a et b) analysent la dynamique de l'emploi dans différents types d'entreprises récemment créées. Ils distinguent les créations originales d'entreprises, les nouvelles entreprises ayant un lien étroit avec les activités principales de l'entreprise mère et les nouvelles créations qui n'ont pas de rapport avec la branche spécifique de l'entreprise mère. Dans cette étude, les créations d'entreprises dont les activités économiques sont en relation étroite avec l'entreprise mère contribuent le plus à la création d'emploi. Mendelson (2000) et Mendelson et Pillai (1999) montrent aussi que la structure d'organisation qui facilite sa capacité de recueillir, analyser et utiliser l'information est aussi responsable du succès de certaines entreprises. Harhoff, Stahl et Woywode (1998) avancent l'argument que la responsabilité juridique d'une entreprise devrait avoir une influence sur le taux de croissance de l'entreprise. Les entrepreneurs fixent, en choisissant la forme juridique, les limites de la responsabilité de l'entreprise. Ces auteurs relèvent que des entreprises à responsabilité limitée possèdent des taux de croissance supérieurs à la moyenne. On peut expliquer cela par le fait que des entrepreneurs, qui ont choisi cette forme, sont plus susceptibles de poursuivre des projets plus risqués ; ceux-ci seraient, en cas de succès, gratifiés par des rendements particulièrement élevés.

Revue internationale P.M.E., vol. 14, $\mathrm{n}^{\text {os }} 3-4,2001$

(C) 2002 - Presses de l'Université du Québec

Édifice Le Delta I, 2875, boul. Laurier, bureau 450, Sainte-Foy, Québec G1V 2M2 • Tél. : (418) 657-4399 - www.puq.uquebec.ca

Tiré de : Revue internationale P.M.E., vol. 14, nos 3-4, sous la direction de Pierre-André Julien. 
Les stratégies des entreprises en matière d'investissement, d'innovation et d'exportation pourraient aussi influencer leur taux de croissance. Les entreprises, devant une demande croissante, doivent augmenter leur capacité de production ; elles vont donc investir dans l'équipement et vraisemblablement augmenter l'emploi. La corrélation entre la hauteur de l'investissement et les créations d'emplois devrait varier avec le rapport optimal entre le capital et le travail dans une branche.

Les modèles théoriques sur les effets de l'innovation sur l'emploi présument souvent que les entreprises qui sont très présentes en recherche et développement possèdent un avantage certain sur leurs concurrents. Les effets de l'innovation sur l'emploi sont pourtant différents : tandis que les innovations réussies de produits suscitent une demande plus importante sur le marché, qui peut se traduire par une croissance positive de l'emploi dans l'entreprise, les innovations en matière de processus, qui ont pour but de réduire les frais, s'accompagnent en général d'une baisse de l'emploi (Katsoulacos, 1986).

Tout comme l'effet des innovations, l'effet sur la croissance de l'entreprise d'une activité importante d'exportation est ambigu. À court terme, des exportations importantes conduisent vraisemblablement à une croissance de l'emploi ; à moyen et à long terme, des exportations soutenues peuvent aussi entraîner la délocalisation de l'emploi vers l'étranger.

Traditionnellement, la diversification est considérée dans la documentation comme une stratégie de croissance. La possibilité d'expansion grâce à une diversification doit toujours être recherchée quand le potentiel interne de croissance d'une entreprise est plus grand que la demande sur les marchés acquis (Penrose, 1995). Dans la documentation récente sur les stratégies de concurrence, la diversification des entreprises a un effet plus nuancé. De plus en plus, les compétences fondamentales d'une entreprise, comme par exemple la capacité à développer et à commercialiser un produit, sont considérées comme la base de ses avantages concurrentiels (Prahalad et Hamel, 1990; Milgrom et Roberts, 1992). De ce point de vue, une diversification latérale, qui ne permettrait pas d'utiliser ou si peu les compétences fondamentales de l'entreprise, ne représente pas une stratégie de croissance adéquate. Une diversification vers un marché dans lequel l'entreprise n'aurait aucune expérience à son actif pourrait avoir des conséquences négatives pour elles.

L'une des causes d'une croissance rapide peut aussi résider dans l'emplacement avantageux d'une entreprise (Steil et Wolf, 1997). Dans les régions rurales, les facteurs de production dont a besoin l'entreprise pour prospérer, comme le personnel qualifié et autres facteurs spécifiques, sont vraisemblablement plus rares que dans les centres urbains. La probabilité de pouvoir bénéficier d'effets d'agglomération est particulièrement élevée en ville; elle augmente avec la proximité d'autres entreprises et d'infrastructures publiques. De plus, les villes possèdent un potentiel de demande supérieur à celui des régions rurales ; il y règne en outre une 
plus grande compétitivité sur les marchés des facteurs de production qui entraîne des prix plus élevés. C'est pourquoi on pourrait supposer que des entreprises qui s'installent en périphérie des villes bénéficient des meilleures conditions possibles et, de ce fait, ont de meilleures chances de grandir plus vite que des entreprises installées en milieux urbains ou ruraux.

3. Dans la documentation sur le management, on accorde un rôle important à l'entrepreneur dans l'explication des différences de croissance entre les entreprises (Finkelstein et Hambrick, 1990 ; Boone, de Brabander et Van Witteloostijn, 1996). Dans ce contexte, le capital humain de l'entrepreneur joue un rôle central. En général, ce capital renvoie à l'âge, à la formation et à l'expérience professionnelle dans la branche. La plupart des études empiriques en arrivent à la conclusion qu'il existe une corrélation positive entre la valeur du capital humain de l'entrepreneur et la performance de l'entreprise (Bates, 1990 ; Brüderl, Preisendörfer et Ziegler, 1992 ; Almus, 2000 ; Brüderl et Preisendörfer, 2000). Des études qui relient les qualités psychologiques de l'entrepreneur à la performance de l'entreprise montrent que, très souvent, des entrepreneurs très axés sur le rendement et très motivés sont à la tête d'entreprises particulièrement performantes. Même si l'observation de telles caractéristiques chez les entrepreneurs est difficile, on ne doit pas oublier que ce sont précisément ces aspects individuels qui peuvent en grande partie expliquer la performance d'une entreprise (Frank et Korunka, 1996). Bien sûr, ce n'est pas seulement le capital humain de l'entrepreneur mais aussi celui des employés qui peut avoir une influence notable sur le succès des entreprises ; ce fait est démontré par les études sur le capital intellectuel des entreprises (Nonaka et Takeuchi, 1997). Dans notre étude, nous n'avons pu traiter de la qualité des employés faute d'informations empiriques.

À ce stade de l'analyse, nous établissons une série d'hypothèses sur les déterminants possibles d'une croissance rapide des entreprises, que nous vérifierons dans le cadre de notre analyse empirique.

H1 : La probabilité pour une entreprise d'appartenir au groupe des entreprises à forte croissance diminue avec la croissance de sa taille.

H2 : Cette probabilité d'appartenir au groupe des entreprises à forte croissance baisse avec l'âge croissant de l'entreprise.

H3 : Les entreprises dépendantes, grâce à leur meilleur accès aux ressources, ont une plus forte probabilité que les indépendantes d'appartenir au groupe des entreprises à croissance rapide.

H4 : La diversification latérale diminue les possibilités de croissance future d'une entreprise.

H5 : Les entreprises à responsabilité limitée ont plus de chances d'appartenir au groupe des entreprises à forte croissance.

Revue internationale P.M.E., vol. 14, $\mathrm{n}^{\text {os }} 3-4,2001$ 
H6: Les entreprises installées à la périphérie des agglomérations urbaines ont une plus forte probabilité de faire partie du groupe des entreprises à forte croissance que celles situées en zone rurale ou à forte concentration urbaine.

H7 : En raison des différences dans les structures de marché et les conditions de concurrence prévalant entre les différentes branches, nous nous attendons, en ce qui concerne le taux de croissance des entreprises, à des variations selon le type de branches industrielles.

H8 : Les dépenses en matière d'innovation, le volume à l'exportation et l'importance des investissements, comme éléments de stratégie de croissance, influent sur la probabilité d'une croissance rapide de l'entreprise.

H9: Plus le capital humain de l'entrepreneur est élevé, plus l'entreprise appartiendra rapidement au groupe des entreprises à forte croissance.

Avant de soumettre ces hypothèses à une analyse économétrique, nous présentons en détail les deux banques de données utilisées, ainsi que les variables les plus importantes.

\section{Sources des données et variables}

\subsection{Base de données}

Deux bases de données séparées, qui contiennent des informations sur un grand nombre d'entreprises de l'Allemagne de l'Ouest pendant une période relativement longue, vont servir à l'examen empirique des hypothèses formulées quant aux déterminants de la forte croissance. Ces deux bases de données, le Mannheimer Unternehmenspanel (MUP) et le Mannheimer Innnovationspanel (MIP) se différencient par leur champ / taille et surtout par la nature des informations qu'elles contiennent sur les entreprises. Alors que le MUP contient un grand nombre d'informations sur les caractéristiques se rapportant aux entreprises et à l'organisation, le MIP contient aussi des informations sur les principaux paramètres stratégiques des entreprises.

\subsubsection{Le Mannheimer Unternehmenspanel (MUP)}

Le Mannheimer Unternehmenspanel (MUP) est une banque qui a été développée à Mannheim au Centre de recherches économiques européennes (ZEW) depuis le mois de juin 1989 par un groupe travaillant sur ce projet. Son objectif est de créer un fichier d'entreprises destiné à observer le développement des entreprises d'Allemagne de l'Ouest au fil du temps. Les données sur les entreprises proviennent de la plus grande agence allemande de crédits CREDITREFORM. Des descriptions détaillées de la structure de la base de données et du traitement de celles-ci se trouvent chez Harhoff, Stahl et Woywode (1998) et chez Woywode (1998).

Revue internationale P.M.E., vol. 14, nºs 3-4, 2001 
Cette base contient des renseignements importants sur les caractéristiques démographiques des entreprises, comme la date de création, la branche, la forme juridique, la composition du capital et du management, le chiffre d'affaires, des informations sur le capital social et sur les rendements du capital. D'autre part, l'agence de crédit donne des indications sur les caractéristiques sociodémographiques des propriétaires et directeurs d'entreprises, dont leur âge, leur sexe, leur situation familiale ainsi que leur diplôme universitaire ou professionnel. Après de multiples corrections, l'échantillon final comprend 8436 entreprises. Comme la taille de l'échantillon et les critères de sélection sont en général de caractère exogène, les estimations ne devraient pas être biaisées par l'exclusion de certaines entreprises.

\subsubsection{Le Mannheimer Innovationspanel (MIP)}

Le Mannheimer Innovationspanel a commencé ses travaux en 1993; il fournit des informations annuelles sur le comportement des entreprises allemandes en matière d'innovation. Les informations sont prélevées par le Centre de recherches économiques européennes (ZEW) en collaboration avec l'Institut pour la recherche sociale appliquée (INFAS). Le projet est soutenu financièrement par le ministère fédéral de l'Éducation, de la Science, de la Recherche et de la Technologie (BMBF). Des descriptions détaillées du MIP se trouvent chez Harhoff et Licht (1994) ainsi que chez Beise et al. (1995). Il est possible de calculer les taux de croissance de l'emploi pour 2136 entreprises du MIP, moins de 59 entreprises dans lesquelles il manque des informations sur au moins une des variables indépendantes utilisées dans les régressions.

\subsection{Description des variables}

Les analyses empiriques évaluent la probabilité pour une entreprise d'appartenir au groupe des $10 \%$ d'entreprises ayant le taux de croissance de l'emploi le plus élevé. La variable explicative dans nos estimations est une variable dichotomique basée sur la distribution des taux de croissance de l'emploi des entreprises observées. C'est pourquoi nous définissons, dans un premier temps, ces taux de croissance, pour ensuite nous attacher à la définition des variables exogènes.

Taux de croissance de l' emploi : Le calcul des taux de croissance de l'emploi s'effectue pour chaque entreprise à partir de la plus longue période d'observation pour laquelle nous disposons d'informations. Afin de standardiser les différentes périodes d'observation, nous utilisons le taux de croissance annuel moyen $\mathrm{Br}$, calculé avec la formule suivante :

$$
\frac{\mathrm{Br}=\log \mathrm{B}\left(t_{2}\right)-\log \mathrm{B}\left(t_{1}\right)}{t_{2}-t_{1}}
$$

Revue internationale P.M.E., vol. 14, $\mathrm{n}^{\text {os }} 3-4,2001$ 
où $\mathrm{B}(t)$ représente le nombre d'employés au temps $t ; t_{1}$ et $t_{2}$ désignent les années pendant lesquelles le nombre d'employés a été relevé. Dans notre analyse, la variable dépendante prend la valeur 1 lorsque l'entreprise concernée affiche un taux de croissance appartenant aux $10 \%$ les plus élevés.

La taille de l' entreprise : la taille de l'entreprise est déterminée par le nombre d'employés au début de l'enquête. Les analyses utilisent le logarithme d'employés (LTAILLE), ainsi que son carré (LTAILLEQ).

L'âge de l' entreprise : l'âge de l'entreprise se calcule par la différence entre la première date de création de l'entreprise et le début de l'enquête. Pour l'estimation, on retient le logarithme de l'âge de l'entreprise (LAGE).

Pour tenir compte de l'éventualité de relations non linéaires entre l'âge de l'entreprise et la croissance de l'emploi, l'âge est aussi intégré dans l'équation sous forme de logarithmes au carré (LAGEQ). De plus, une interaction entre l'âge logarithmé et la taille logarithmée (LAGE*LTAILLE) est ajoutée à l'équation.

Le degré d' indépendance de l' entreprise : le degré d'indépendance de l'entreprise résulte de la situation des entreprises en termes de participations. Une entreprise est entièrement dépendante d'une autre entreprise lorsqu'une ou plusieurs autres entreprises détiennent ensemble $100 \%$ des participations. La variable dichotomique FILIALE prend la valeur de 1 lorsque l'entreprise appartient à $100 \%$ à une ou plusieurs entreprises et la valeur 0 dans les autres cas.

Le degré de diversification: le degré de diversification d'une entreprise est indiqué à l'aide d'une variable dichotomique (DIVERS). Cette variable prend la valeur de 1 lorsque les données du CREDITREFORM attribuent à l'entreprise deux branches d'activité ou plus considérées comme différentes au regard de la nomenclature établie par l'office fédéral des statistiques.

La forme juridique : la forme juridique est introduite sous forme de variables dichotomiques dans l'équation. Dans ce travail, les formes juridiques suivantes sont distinguées : 1) entreprise unipersonnelle à responsabilité limitée (EURL), 2) société civile (SC), 3) société en nom collectif (SNC), 4) société en commandite simple (SCS), 5) société à responsabilité limitée (SARL), 6) SARL SCS ainsi que 7) la société anonyme (SA). Le groupe des entreprises unipersonnelles (EURL), dans lequel on réunit entreprise unipersonnelle et petites ou moyennes entreprises, sert de catégorie de base. Pour éviter les éventuels problèmes de simultanéité lors de l'estimation, nous utilisons la forme juridique que l'entreprise possédait au $1^{\mathrm{er}}$ juillet $1989^{1}$.

1. Pendant la période d'observation de juin 1989 à mars 1996, seulement 3,2\% des entreprises ont changé de forme juridique. Dans la plupart des cas, les entreprises concernées étaient des sociétés de personnes et sont devenues des sociétés de capitaux. 
La branche économique: des variables dichotomiques ont été créées pour les branches d'activité. Ces variables contrôlent les effets spécifiques à l'industrie selon la nomenclature à un ou deux chiffres des secteurs d'activité de l'office fédéral des statistiques.

Les dépenses pour l' innovation : pour notre échantillon MIP, nous calculons la part des dépenses consacrées à la recherche et le développement par rapport au chiffre d'affaires de l'année de base (INNOV). De plus, la part du personnel en R-D est déterminée de façon similaire par rapport au nombre total des employés (R-D).

La hauteur des investissements et le taux d' exportation: pour les entreprises de l'échantillon MIP, deux autres dimensions de leur stratégie sont analysées qui expriment leur propension à l'investissement et à l'exportation. La variable INVEST donne le rapport de l'investissement sur le chiffre d'affaires total (INVEST) pour l'année de base. La variable INVESTQ en est le carré. L'intensité des exportations (EXINT) est calculée comme la part du chiffre d'affaires à l'exportation dans le chiffre d'affaires total pendant l'année de base des taux de croissance. En outre, nous créons une variable dichotomique (EXPO50), qui prend la valeur de 1 lorsque la part des exportations représente plus de $50 \%$ du chiffre d'affaires. Dans l'équation estimée est introduite une variable combinée supplémentaire, EXPO50*EXINT.

Le lieu d' implantation de l' entreprise : nous distinguons trois sortes d'emplacement : 1) les agglomérations urbaines (URBAIN), 2) les emplacements suburbains (SUBURB) et 3 ) les régions rurales (LAND ou CAMPAGNE). Le classement des régions dans les trois types d'emplacement se fait selon une classification développée par Seitz (1996); les entreprises en régions rurales servent de groupe de référence.

Le capital humain de l' entrepreneur : le capital humain de l'entrepreneur est mesuré grâce à deux indicateurs : d'une part, son âge au début de l'enquête et, d'autre part, son plus haut diplôme universitaire ou professionnel. Dans le cas où plusieurs propriétaires ou entrepreneurs existent, on utilise l'âge ou bien le diplôme universitaire ou professionnel du propriétaire qui possède la plus grande part du capital propre de l'entreprise. L'âge de l'entrepreneur est introduit en termes loglinéaire (LPERALT) (LPERAGE) dans l'équation. Le diplôme le plus élevé de formation de l'entrepreneur est déterminé aussi sur la base des informations concernant les personnes du MUP. Les entrepreneurs sont différenciés selon leur diplôme universitaire. La variable dichotomique AKAD (ACAD) prend la valeur de 1 lorsqu'un entrepreneur détient un diplôme universitaire, sinon 0.

Les variables dichotomiques selon la période: afin d'obtenir des évaluations fiables, la période d'observation sur laquelle on a calculé les taux de croissance est contrôlée par des variables dichotomiques.

Revue internationale P.M.E., vol. 14, nos 3-4, 2001

(C) 2002 - Presses de l'Université du Québec

Édifice Le Delta I, 2875, boul. Laurier, bureau 450, Sainte-Foy, Québec G1V 2M2 • Tél. : (418) 657-4399 - www.puq.uquebec.ca

Tiré de : Revue internationale P.M.E., vol. 14, nos 3-4, sous la direction de Pierre-André Julien. 


\section{Résultats empiriques}

Pour la partie empirique du travail, nous faisons un retour sur les deux banques de données pour faire ressortir la différence entre le MIP et le MUP. Grâce à la présentation des quantiles des taux de croissance de l'emploi, nous allons illustrer la dispersion des performances des entreprises en termes de croissance. Nous démontrerons par la suite l'importance des entreprises à forte croissance pour l'emploi et pour l'économie en général. La section se terminera par l'analyse économétrique des facteurs qui aideront à identifier les caractéristiques des entreprises qui augmentent la probabilité d'appartenir au groupe des gazelles.

\subsection{Résultats descriptifs}

Dans le tableau 1 se trouvent des données descriptives sur les entreprises des deux échantillons. À ce propos, il faut rappeler que les entreprises provenant aussi bien du MUP que du MIP ont été choisies selon des critères de tirage bien précis. Les statistiques descriptives ne sont donc en aucun cas représentatives de toutes les entreprises ouest-allemandes.

TABLEAU 1

Statistiques descriptives des contrôles utilisés

\begin{tabular}{lcccc}
\hline & \multicolumn{2}{c}{ MUP } & \multicolumn{2}{c}{ MIP } \\
& \multicolumn{1}{c}{$(\mathbf{1 9 8 9 - 1 9 9 5 )}$} & \multicolumn{2}{c}{$(\mathbf{1 9 9 2 - 1 9 9 5 )}$} \\
\hline Variable & Moyenne & Écart-type & Moyenne & Écart-type \\
\hline Taux de croissance de l'emploi & 0,02 & 0,21 & $-0,02$ & 0,35 \\
Taux de croissance du chiffre d'affaires & 0,10 & 0,31 & 0,02 & 0,40 \\
\hline Nombre de salariés (TAILLE) & 276,97 & 2258,01 & 1087,03 & 7235,21 \\
Médiane (TAILLE) & 8 & - & 159 & - \\
Âge de l'entreprise (AGE) & 29,20 & 35,31 & 45,16 & 44,21 \\
\hline SC & 0,02 & 0,14 & - & - \\
SNC & 0,02 & 0,13 & 0,01 & 0,12 \\
SCS & 0,04 & 0,20 & 0,04 & 0,20 \\
SARL SCS & 0,10 & 0,30 & 0,26 & 0,44 \\
SARL & 0,34 & 0,48 & 0,54 & 0,50 \\
SA & 0,04 & 0,20 & 0,08 & 0,27 \\
\hline
\end{tabular}

Les statistiques descriptives montrent clairement que les entreprises des deux échantillons sont fondamentalement différentes. L'entreprise moyenne dans le MUP est beaucoup plus petite que celle du MIP et plus jeune d'environ 15 ans. Près de $80 \%$ des entreprises MIP, mais seulement environ $50 \%$ des entreprises MUP sont inscrites dans le registre du commerce et des sociétés (RCS). La moyenne des taux de croissance de l'emploi diffère aussi selon l'échantillon. De 1989 à 1995, le taux 
de croissance moyen des entreprises MUP était positif. Dans le cas du MIP, entre 1992 et 1995 , le taux de croissance moyen est négatif de $-0,2 \%$; on peut en conclure que les entreprises de cette banque ont perdu en moyenne des emplois pendant cette période.

Nous analysons tout d'abord la répartition des taux de croissance de l'emploi dans les deux banques de données. De plus, nous calculons les taux de croissance pour différentes périodes.

TABLEAU 2

Distribution des taux de croissance

\begin{tabular}{|c|c|c|c|c|c|}
\hline \multirow[t]{2}{*}{ Quantile } & \multicolumn{3}{|c|}{$\begin{array}{c}\text { MUP } \\
\text { Période }\end{array}$} & \multicolumn{2}{|c|}{$\begin{array}{c}\text { MIP } \\
\text { Période }\end{array}$} \\
\hline & 90-92 & 93-95 & $90-95$ & $92-93$ & 93-95 \\
\hline $1 \%$ & $-62,78 \%$ & $-95,45 \%$ & $-53,81 \%$ & $-179,18 \%$ & $-8,75 \%$ \\
\hline $5 \%$ & $-17,40 \%$ & $-25,02 \%$ & $-15,12 \%$ & $-47,00 \%$ & $-25,88 \%$ \\
\hline $10 \%$ & $-5,93 \%$ & $-13,81 \%$ & $-8,11 \%$ & $-26,24 \%$ & $-18,63 \%$ \\
\hline $25 \%$ & $0,00 \%$ & $-3,10 \%$ & $-2,06 \%$ & $-11,78 \%$ & $-7,04 \%$ \\
\hline $50 \%$ & $0,00 \%$ & $0,00 \%$ & $0,00 \%$ & $-\quad 2,52 \%$ & $-0,81 \%$ \\
\hline $75 \%$ & $5,23 \%$ & $4,87 \%$ & $5,30 \%$ & $3,28 \%$ & $4,63 \%$ \\
\hline $90 \%$ & $17,98 \%$ & $12,79 \%$ & $11,72 \%$ & $19,42 \%$ & $12,57 \%$ \\
\hline $95 \%$ & $30,49 \%$ & $23,16 \%$ & $17,24 \%$ & $51,08 \%$ & $25,54 \%$ \\
\hline $99 \%$ & $60,20 \%$ & $53,42 \%$ & $31,14 \%$ & $185,63 \%$ & $64,46 \%$ \\
\hline Moyenne & $2,79 \%$ & $-1,23 \%$ & $1,02 \%$ & $-\quad 2,85 \%$ & $-1,18 \%$ \\
\hline Observations & 3,680 & 3,644 & 3,308 & 962 & 788 \\
\hline
\end{tabular}

Comme nous pouvons le voir au tableau 2, la médiane des taux de croissance de l'emploi (quantile à $50 \%$ ) se situe à $0 \%$ dans le MUP (tous secteurs confondus) pour les trois périodes observées. En ce qui concerne le MIP (secteur secondaire, industrie de transformation), la médiane des taux de croissance de l'emploi pour la période 1992-1995 est négative. Pendant la période 1990-1992, une entreprise devait avoir un taux de croissance supérieur à $18 \%$ pour appartenir au groupe des entreprises à forte croissance. Entre 1993 et 1995, les taux de croissance ne devaient se situer qu'au-delà de $13 \%$ pour atteindre le décile supérieur des taux de croissance supérieurs dans les deux banques de données. Selon les résultats de nos enquêtes, la moyenne des taux de croissance de l'emploi a diminué dans la première moitié des années 1990. La conjoncture économique favorable au début des années 1990 du fait de la réunification allemande, dont ont profité beaucoup d'entreprises ouestallemandes, s'est dégradée dans les années suivantes. À partir de 1992-1993, l'économie allemande se trouvait dans une phase de récession. Pendant toute la période de 1990 à 1995, les entreprises du MUP devaient croître de plus de 12 \% pour, selon notre définition, faire partie des entreprises à croissance rapide. Ce résultat indique 
qu'il est difficile pour une entreprise de connaître, sur une longue période, une croissance élevée de l'emploi. C'est ce qui ressort également de la comparaison des taux de croissance dans le MIP, qui sont plus bas dans les déciles supérieurs sur la plus longue période de 1993 à 1995 que sur la brève période 1992-1993; cela, alors que la médiane des taux de croissance était plus élevée sur la plus longue période que sur la plus brève de 1992 à 1993.

D'après ce tableau, il est évident qu'au moins un quart des entreprises des deux banques de données ont perdu des emplois alors que quelques-unes n'ont pas modifié leurs effectifs; seules $10 \%$ d'entre elles environ ont réalisé une croissance de l'emploi à deux chiffres. De cette variation considérable, en termes de performance, on peut comprendre l'impact de ces entreprises à forte croissance sur l'emploi général. Cela apparaît clairement au tableau 3 du MUP, qui présente le développement de l'emploi en général. Nous rappelons que ces données, n'ayant pas été calculées sur toutes les entreprises allemandes, ne sont donc pas représentatives de l'Allemagne de l'Ouest. Le tableau 3 illustre plutôt, grâce aux modifications à l'intérieur d'un contrôle, l'importance des entreprises à forte croissance.

TABLEAU 3

Contributions à l'emploi des entreprises MUP de 1990-1995

\begin{tabular}{lcccc}
\hline Entreprises & Nombre & \multicolumn{2}{c}{ Emploi } & Différence \\
& $\boldsymbol{\%}$ & $\mathbf{1 9 9 0}$ & $\mathbf{1 9 9 5}$ & \\
\hline En diminution & 1,011 & $1,406,847$ & 934,697 & $-472,149$ \\
Stagnante & $31 \%$ & $62 \%$ & $44 \%$ & \\
& 831 & 83,406 & 82,406 & 0 \\
En croissance & $25 \%$ & $4 \%$ & $4 \%$ & \\
& 1,135 & 711,91 & 856,965 & 145,874 \\
En forte croissance & $34 \%$ & $31 \%$ & $41 \%$ & \\
& 331 & 83,760 & 232,229 & 148,470 \\
\hline Total & $10 \%$ & $4 \%$ & $11 \%$ & \\
\hline
\end{tabular}

Dans l'ensemble, le tableau 3 montre que l'emploi a diminué au fil du temps dans les entreprises pérennes en 1990 et en 1995. Presque un tiers des entreprises ont licencié des employés; $62 \%$ des employés ont été touchés. Un quart des entreprises, qui représente seulement $4 \%$ des employés, ont stagné et un autre tiers ont crû de façon modérée. Par définition seulement, $10 \%$ font partie des entreprises à forte croissance. Ce petit groupe d'entreprises a créé, au début des années 1990, plus d'emplois que le groupe trois fois plus important des entreprises en croissance! Pendant cette période de croissance, ce petit groupe a pu pratiquement tripler sa contribution à l'emploi pour atteindre $11 \%$. Derrière ces chiffres se cache une 
dynamique considérable d'augmentation et de réduction d'emplois, dans laquelle un très petit groupe d'entreprises sont responsables dans une large mesure de nouveaux emplois.

C'est pour cette raison que les entreprises à forte croissance sont très importantes pour la politique de l'emploi. Nous examinerons par la suite en quoi ce groupe se différencie des autres entreprises dans l'analyse économétrique.

\subsection{Modèles de probit pour l'estimation de la probabilité d'une forte croissance de l'emploi}

Les résultats des différents modèles de probit vont être présentés d'abord sur la base de données tirées du MUP puis sur celle du MIP. Nous estimons la probabilité que les entreprises fassent partie du groupe des gazelles en fonction d'un certain nombre de variables. Nos estimations se composent de trois modèles différents que nous essayons de spécifier de façon aussi comparable que possible pour le MUP et le MIP.

\subsubsection{Résultats de l'estimation sur la base du MUP}

Pour tester nos hypothèses sur la base du MUP, les deux premiers modèles intègrent toutes les variables sauf celles touchant le capital humain de l'entrepreneur. Le premier modèle comprend cinq variables binomiales décrivant la branche d'activité, dont la branche de référence est l'industrie de transformation. En comparaison, nous utilisons, dans le deuxième modèle, 28 branches économiques. Le troisième modèle comprend enfin deux variables supplémentaires qui tiennent compte du capital humain de l'entrepreneur: son âge et son niveau de formation. En ce qui concerne les sociétés anonymes et les entreprises dépendantes, on ne peut préciser les personnes naturelles comme propriétaires, c'est pourquoi on ne peut pas faire entrer dans les calculs les indicateurs de l'âge et de formation. Ces entreprises seront exclues de notre analyse pour le troisième modèle. Dans le tableau 4, nous présentons les résultats des estimations.

Comme nous le voyons au tableau 4, il semble exister, dans les trois modèles, une relation non linéaire entre la taille de l'entreprise et la probabilité de forte croissance de l'emploi. Pour être plus précis, cette probabilité diminue dans un premier temps avec la taille, mais elle réaugmente à partir d'une certaine taille. D'après les données dont nous disposons, nous ne pouvons pas savoir si une entreprise réalise une croissance de l'emploi à l'interne ou à l'externe. Les très grandes entreprises ont vraisemblablement de plus fortes chances de croître grâce à de nouvelles acquisitions que les petites; ces dernières en revanche connaissent surtout une croissance interne.

Nous avons trouvé une relation négative entre l'âge de l'entreprise et la probabilité d'appartenir au groupe de gazelles. Cet effet est significatif pour les deux

Revue internationale P.M.E., vol. 14, $\mathrm{n}^{\text {os }} 3-4,2001$ 
TABLEAU 4

Résultats de l'estimation de modèle de probit pour le MUP

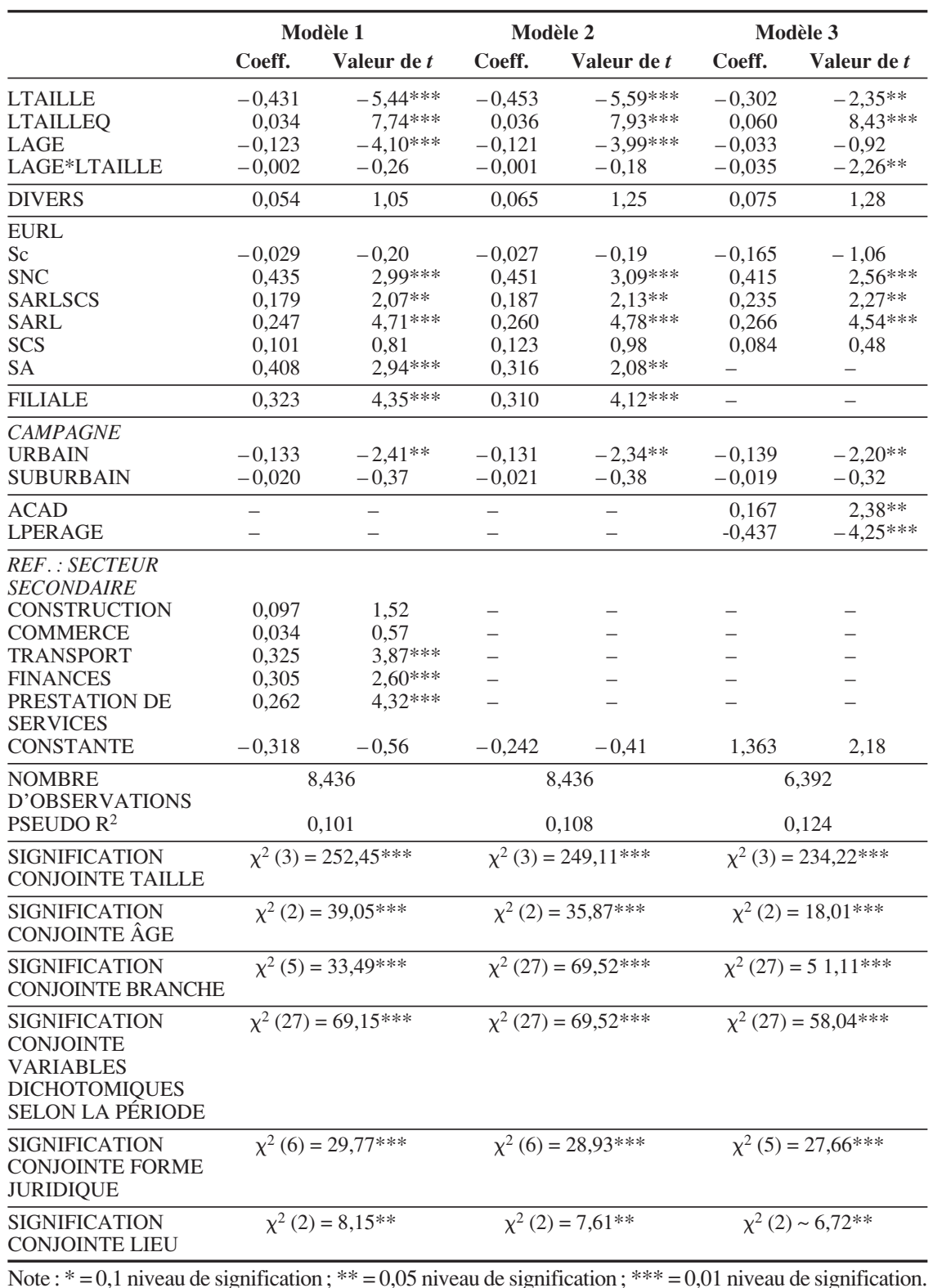


premiers modèles, mais il ne l'est pas dans le troisième modèle, comprenant l'âge du propriétaire. Cependant, les tests $\mathrm{F}$ communs aux paramètres de l'âge et de la taille sont significatifs au niveau de $1 \%$.

En accord avec les hypothèses présentées à la section 1, les effets de la forme juridique sur la probabilité d'une croissance rapide sont significatifs. Les sociétés à responsabilité limitée, tout comme les sociétés en nom collectif, se développent plus vite que les entreprises individuelles. La probabilité d'appartenir au groupe d'entreprises à forte croissance dépend aussi de la branche. Les résultats qui ressortent du premier modèle montrent que les entreprises des secteurs du transport, des finances et de l'assurance, et des services ont une plus forte chance de croître que celles de l'industrie de transformation. Dans le deuxième modèle, 28 branches économiques ont été utilisées; elles sont significatives au niveau de $1 \%$ d'après le test de significativité conjointe.

À la première section, nous avons formulé l'hypothèse que la diversification latérale ralentit la croissance de l'entreprise et que la probabilité que des entreprises diversifiées appartiennent au groupe des gazelles diminue d'autant. Les résultats de nos analyses empiriques ne confirment pas cette hypothèse. En effet, nous ne pouvons établir de rapport significatif entre la diversification et une croissance forte de l'emploi.

Les filiales possèdent une plus forte probabilité de faire partie des entreprises à forte croissance que les firmes indépendantes. Ce résultat confirme notre hypothèse selon laquelle les entreprises dépendantes réalisent une croissance de l'emploi particulièrement élevée grâce à un meilleur accès aux ressources telles que le capital et le savoir-faire. En outre, nous pouvons établir un lien entre l'emplacement de l'entreprise et sa croissance. Les entreprises qui se sont implantées dans des agglomérations urbaines possèdent une probabilité plus faible d'appartenir au groupe des entreprises à croissance rapide que celles en région suburbaine.

Les caractéristiques de l'entrepreneur influent aussi sur la probabilité d'une forte croissance; cette probabilité diminue avec l'âge de l'entrepreneur. Vraisemblablement, les jeunes entrepreneurs font preuve d'une grande motivation et d'un fort engagement lorsque l'économie le permet; cet engagement semble se perdre avec le vieillissement de l'entrepreneur. La formation universitaire semble aussi avoir un effet positif sur la croissance. Une interprétation possible est qu'un niveau élevé de formation universitaire favorise la productivité et la crédibilité de l'entrepreneur. Ceci aiderait par exemple l'entrepreneur à surmonter des restrictions financières existantes.

Les variables dichotomiques annuelles sont fortement significatives dans les trois estimations. Apparemment, la période d'observation pendant laquelle les taux de croissance ont été calculés a une influence importante sur la force des taux de croissance. Nous n'allons toutefois pas discuter de ces variables dichotomiques par année.

Revue internationale P.M.E., vol. 14, nos 3-4, 2001 


\subsection{Résultats de l'estimation sur la base du MIP}

Nous allons maintenant présenter les résultats des trois modèles de probit sur la base des données du MIP de façon analogue à l'estimation sur la base de MUP. De nouveau, nous évaluons la probabilité pour une entreprise d'appartenir au groupe des entreprises à forte croissance. Le premier modèle est notre modèle de base ; il comprend les variables de la taille, l'âge, la branche et la forme juridique de l'entreprise comme variables explicatives. Le modèle 2 inclut trois variables de plus, lesquelles spécifient le lieu d'implantation de l'entreprise. De plus, nous étudions dans le modèle 3 les effets de différents aspects de la stratégie d'une entreprise sur ses chances de croissance, comme par exemple son comportement à l'égard de l'exportation, de l'investissement ou son activité dans le sens de l'innovation.

Dans le tableau 5, nous présentons les résultats de l'estimation. De nouveau, nous avons utilisé comme référence de l'évaluation les taux de croissance de l'emploi établis sur la plus longue période et nous les avons contrôlés à l'aide de variables dichotomiques selon la période pour différentes années de départ et différentes périodes.

Nous nous contenterons ici de parler des divergences essentielles par rapport aux résultats du tableau 4 et de discuter des nouveaux résultats. L'influence de la taille de l'entreprise sur la probabilité d'appartenir au groupe à forte croissance est, pour les trois modèles, presque identique aux résultats obtenus par le MUP, même s'ils ne sont pas aussi significatifs que les résultats correspondant au tableau 4. L'effet de l'âge de l'entrepreneur sur la probabilité de croissance n'est significatif que dans la troisième estimation. Il existe ici un effet non linéaire de l'âge, négatif pour $90 \%$ de l'échantillon.

Les résultats de l'estimation concernant l'influence de la forme juridique sur la croissance des entreprises confirment en grande partie nos prévisions. Les coefficients pour les SARL et SCS tout comme pour les SA sont positifs et significatifs. Le coefficient de la SARL est certes positif dans les trois modèles, mais non significatif.

Dans les estimations du MIP qui, comme nous l'avons dit auparavant, se limitent aux entreprises de l'industrie de transformation et des services proches de la production, nous ne pouvons prouver de différences significatives concernant la probabilité de croissance entre les différentes branches économiques. Dans le test de significativité globale, les variables dichotomiques par industrie sont non significatives. Vraisemblablement, les conditions du marché comme le développement de la demande ou la taille minimale optimale de l'entreprise sont trop semblables dans les branches de l'industrie de transformation pour qu'il en résulte des conséquences durables spécifiques aux branches sur la probabilité de croissance. C'est pourquoi l'appartenance aux branches, dans les évaluations du MIP, n'apporte pas de contribution significative à nos explications.

Revue internationale P.M.E., vol. 14, nºs 3-4, 2001 
TABLEAU 5

Résultats probit de l'évaluation pour le MIP

\begin{tabular}{|c|c|c|c|c|c|c|}
\hline & \multicolumn{2}{|c|}{ Modèle 1} & \multicolumn{2}{|c|}{ Modèle 2} & \multicolumn{2}{|c|}{ Modèle 3} \\
\hline & Coeff. & Valeur de $t$ & Coeff. & Valeur de $t$ & Coeff. & Valeur de $t$ \\
\hline LTAILLE & $-0,304$ & $-3,31 * * *$ & $-0,300$ & $-3,23 * * *$ & $-0,243$ & $-2,28 * *$ \\
\hline LTAILLEQ & 0,027 & $3,21 * * *$ & 0,026 & 3,11 & 0,023 & $2,46^{* *}$ \\
\hline LAGE & 0,185 & 0,97 & 0,166 & 0,85 & 0,276 & \\
\hline \multicolumn{7}{|l|}{1,33} \\
\hline LAGEQ & $-0,017$ & $-0,60$ & $-0,015$ & $-0,52$ & $-0,032$ & $-1,04$ \\
\hline LAGE*LTAILLE & $-0,028$ & $-1,45$ & $-0,028$ & $-1,44$ & $-0,034$ & $-1,51$ \\
\hline \multicolumn{7}{|l|}{ RÉF.CONSTRUCTION } \\
\hline ÉNERGIE & $-0,510$ & $-1,70 *$ & $-0,522$ & $-1,75^{*}$ & $-0,829$ & $-2,40$ \\
\hline CHIMIE & $-0,080$ & $-0,47$ & $-0,097$ & $-0,56$ & $-0,118$ & $-0,64$ \\
\hline CAOUTCHOUC & 0,124 & 0,75 & 0,165 & 0,98 & 0,104 & 0,56 \\
\hline MATIÈRES PREMIÈRES & S 1,149 & 0,82 & 0,213 & 1,16 & 0,210 & 1,05 \\
\hline MÉTALLURGIE & $-0,228$ & $-1,26$ & $-0,225$ & $-1,22$ & $-0,254$ & $-1,26$ \\
\hline ÉLECTROTECHNIQUE & 0,144 & 1,20 & 0,150 & 1,24 & 0,108 & 0,83 \\
\hline AUTRES & $-0,072$ & $-0,55$ & $-0,056$ & $-0,42$ & $-0,032$ & $-0,22$ \\
\hline CONSTRUCTION & 0,236 & 1,23 & 0,210 & 1,10 & 0,092 & 0,42 \\
\hline PRESTATION DE & 0,284 & $1,75^{*}$ & 0,261 & 1,59 & $-0,134$ & $-0,48$ \\
\hline \multicolumn{7}{|l|}{ SERVICE } \\
\hline \multicolumn{7}{|l|}{ RÉF PROPRIÉTAIRE } \\
\hline SARLSCS & 0,392 & $2,18 * *$ & 0,404 & $2,24 * *$ & 0,336 & 1,64 \\
\hline SCS & $-0,200$ & $-0,70$ & $-0,149$ & $-0,52$ & $-0,051$ & $-0,17$ \\
\hline SARL & 0,22 & 1,32 & 0,220 & 1,28 & 0,178 & 0,90 \\
\hline SA & 0,791 & $3,38 * *$ & 0,728 & $3,11 * *$ & 0,636 & 2,39 \\
\hline \multicolumn{7}{|l|}{$\overline{R E F} . C A M P A G N E$} \\
\hline URBAIN & - & - & 0,261 & $2,27 * *$ & 0,356 & 3,04 \\
\hline SUBURBAIN & - & - & 0,139 & 1,32 & 0,178 & 1,54 \\
\hline INNOVATION & - & - & - & - & 0,027 & 0,44 \\
\hline R-D & - & - & - & - & 1,074 & 2,42 \\
\hline INVESTISSEMENT & - & - & - & - & 1,140 & 2,85 \\
\hline INVESTSQ & - & - & - & - & $-0,222$ & 2,63 \\
\hline EXPO50 & - & - & - & - & 1,511 & 2,07 \\
\hline EXPO50*EXINT & - & - & - & - & $-2,293$ & $-2,08$ \\
\hline CONSTANTE & $-0,647$ & $-1,44$ & $-0,532$ & $-1,05$ & $-1,148$ & $-2,24$ \\
\hline \multirow{3}{*}{$\begin{array}{l}\text { NOMBRE } \\
\text { D'OBSERVATIONS } \\
\text { PSEUDO R }{ }^{2}\end{array}$} & \multicolumn{2}{|r|}{2,077} & \multicolumn{2}{|r|}{2,077} & \multicolumn{2}{|r|}{1,833} \\
\hline & \multirow{2}{*}{\multicolumn{2}{|c|}{0,063}} & \multirow{2}{*}{\multicolumn{2}{|c|}{0,073}} & \multirow{2}{*}{\multicolumn{2}{|c|}{0,088}} \\
\hline & & & & & & \\
\hline SIGNIFICATION & \multirow{2}{*}{\multicolumn{2}{|c|}{$\chi^{2}(3)=33,88^{* *}$}} & \multirow{2}{*}{\multicolumn{2}{|c|}{$\chi^{2}(3)=33,49 * *$}} & \multirow{2}{*}{\multicolumn{2}{|c|}{$\chi^{2}(3)=20,61^{* *}$}} \\
\hline CONJOINTE TAILLE & & & & & & \\
\hline $\begin{array}{l}\text { SIGNIFICATION } \\
\text { CONJOINTE ÂGE }\end{array}$ & \multicolumn{2}{|c|}{$\chi^{2}(3)=5,49$} & \multicolumn{2}{|c|}{$x^{2}(3)=5,86$} & \multicolumn{2}{|c|}{$\chi^{2}(3)=7,92^{* *}$} \\
\hline SIGNIFICATION & \multirow{2}{*}{\multicolumn{2}{|c|}{$\chi^{2}(9)=14,56$}} & $\chi^{2}(9$ & $=14,81^{*}$ & $\chi^{2}(C$ & $=12,32$ \\
\hline CONJOINTE BRANCHE & & & & & & \\
\hline SIGNIFICATION & $x^{2}(4$ & $=19,54 * *$ & $\chi^{2}(4$ & $=16,91 * *$ & $\chi^{2}(4$ & $=9,81 * *$ \\
\hline CONJOINTE FORME & & & & & & \\
\hline 5 & & & & & & \\
\hline SIGNIFICATION & & - & $x^{2}(2$ & $=5,17^{*}$ & $x^{2}(2$ & $=9,48 * *$ \\
\hline CONJOINTE LIEU & & & & & & \\
\hline
\end{tabular}

Revue internationale P.M.E., vol. 14, $\mathrm{n}^{\text {os }} 3-4,2001$ 
Les résultats sur le rôle du lieu d'implantation dans la croissance de l'entreprise contredisent nos prévisions et surtout les résultats du MUP. Alors que nous supposions que les entreprises en régions suburbaines se développent plus rapidement, il semble ici que ce soient les entreprises en agglomérations urbaines qui se caractérisent par une forte croissance. Le coefficient des régions suburbaines est certes positif dans les estimations, mais non significatif.

À côté des facteurs nommés ci-dessus, nous avons, dans la partie théorique du travail, présenté l'argument que la stratégie de l'entreprise devrait se répercuter clairement sur sa performance en termes de croissance. Dans le cas présent, nous supposions que le niveau des investissements, l'importance des exportations et l'ampleur des activités de R-D influent sur la probabilité de réaliser une forte croissance. L'importance de la stratégie pour la croissance va être étayée par le constat que l'inclusion des variables reflétant la stratégie de l'entreprise fait augmenter de plus de $1 \%$ le pseudo-R_dans le troisième modèle. Le poids des variables dichotomiques par industrie baisse dans le modèle 3 par rapport au premier et au deuxième modèle. Les variables dichotomiques par industrie reflètent peut-être les effets des investissements, de l'exportation et des activités en R-D.

La part des dépenses consacrées à l'innovation par rapport au chiffre d'affaires total (INNOV) n'influence pas la probabilité d'appartenir au groupe à croissance élevée. Par contre, l'intensité en R-D, mesurée par la part du personnel en R-D par rapport au chiffre total des employés, est significativement et positivement corrélée avec la probabilité de forte croissance. La corrélation entre les deux variables de l'innovation n'est que de 0,03. La variable R-D est peut-être un meilleur indicateur des innovations produits plus porteuses d'emplois que la variable (INNOV), qui englobe les innovations en termes de processus tout comme les réalisations dans la recherche et le développement tirées de l'extérieur.

L'effet marginal du montant des investissements par rapport au chiffre d'affaires est opérationnalisé par une fonction linéaire et une fonction carrée. Dans l'intervalle considéré, une augmentation marginale de l'indice augmente la probabilité d'appartenir au groupe d'entreprises à forte croissance, avec toutefois un taux en diminution. Comme les investissements et le chiffre d'affaires indiqués ont été déterminés sur l'année de base du taux de croissance, nous concluons qu'il existe entre le comportement à l'égard de l'investissement et le développement de l'emploi une relation positive; mais, bien sûr, l'emploi suit les investissements avec un décalage dans le temps.

Nous pouvons prouver une influence non linéaire du taux d'exportation sur la probabilité d'une croissance rapide de l'entreprise. Tout d'abord, il est judicieux de distinguer les entreprises avec un taux d'exportation supérieur ou inférieur à $50 \%$. D'une part, les entreprises qui réalisent $50 \%$ de leurs chiffres d'affaires à l'exportation possèdent une probabilité beaucoup plus élevée de croître que les 
autres. D'autre part, il apparaît que pour les entreprises qui enregistrent plus de $50 \%$ de leurs chiffres d'affaires à l'étranger, la probabilité d'une forte croissance baisse de façon significative avec la part de l'exportation. Ce résultat porte à croire que les entreprises fortement exportatrices ont tendance à investir directement à l'étranger et à y embaucher du personnel, alors qu'elles diminuent les emplois à l'intérieur du pays.

Enfin, les variables dichotomiques selon la période, qui contrôlent les périodes d'observation, sont significatives; ce qui laisse penser que, pendant la période d'observation entre 1992 et 1995, les conditions générales de l'économie ont changé, ce qui s'est répercuté sur les chances de croissance.

\section{Conclusion}

Bien que les déterminants de la croissance de l'entreprise aient été souvent étudiés par le passé, les études sur la forte croissance des entreprises sont encore rares. Les quelques études existantes se retrouvent dans les travaux en management (Kanter, 1994 ; Peters et Waterman, 1994 ; Peters, 1998 ; Simon, 1996 ; Kim et Mauborgne, 1997). Seules les entreprises prospères, à part quelques exceptions, y sont analysées; cela limite le caractère explicatif de ces études. Même des études aussi éminentes que Europe's 500, une étude sur les 500 entreprises européennes les plus prospères, souffrent de ce «biais ». On peut facilement critiquer les résultats de ces travaux.

Par contraste, notre étude examine les différences systématiques entre les entreprises à forte croissance et les entreprises à croissance plus lente. Plus précisément, nous analysons l'impact de facteurs liés à l'entreprise ainsi qu'à l'entrepreneur. Les effets des branches sont contrôlés par des variables dichotomiques. Selon des études récentes, la probabilité d'une forte croissance est influencée par la taille et l'âge de l'entreprise. Il existe, entre la taille de l'entreprise et la probabilité de forte croissance, un rapport inverse en forme de $\mathrm{U}$, ce qui signifie que les petites et les très grandes entreprises possèdent une probabilité particulièrement élevée de se développer. Nous observons un effet négatif de l'âge de l'entreprise sur la probabilité de croissance rapide des entreprises. Nos résultats indiquent aussi que les entreprises dont la forme juridique est à responsabilité limitée ont plus de chances de croître. Les entreprises dépendantes, comme les filiales, se développent aussi plus vite que les entreprises indépendantes.

Nous pouvons affirmer que les caractéristiques de l'entrepreneur, comme son âge ou sa formation, contribuent de façon essentielle à l'explication de la croissance. Les entreprises à forte croissance sont très souvent dirigées par de jeunes entrepreneurs. On peut, à ce propos, voir l'âge de l'entrepreneur comme un indicateur de sa motivation et sa disposition à orienter son entreprise vers la croissance. L'indicateur

Revue internationale P.M.E., vol. 14, $\mathrm{n}^{\text {os }}$ 3-4, 2001 
du capital humain, que nous avons utilisé sous la forme du plus haut diplôme de fin d'études, représente un premier pas pour rendre opérationnel le concept de capital humain. L'expérience dans le secteur d'activité, dans le management ou bien dans une activité ancienne autonome semblent être des éléments intéressants touchant le capital humain par rapport aux déterminants de la forte croissance. Malheureusement, ces variables ne sont pas disponibles dans les banques de données utilisées. Nous aurions aussi préféré inclure des informations sur le capital humain des employés, car nous croyons qu'un niveau élevé de capital intellectuel des employés représente un avantage concurrentiel. Ces variables ne sont pas non plus disponibles dans les banques de données utilisées.

Finalement, la stratégie de l'entreprise se révèle un facteur important d'explication de la forte croissance. Nous pouvons établir, pour les entreprises du MIP, que les activités d'investissement et d'innovation, tout comme l'ampleur des exportations influent de façon significative sur la probabilité de croissance. Notons également que les entreprises du domaine des services possèdent une plus forte probabilité de connaître une croissance rapide de l'emploi que les entreprises des autres secteurs.

Bien que nos deux banques de données contiennent un grand nombre de variables explicatives concernant la croissance des entreprises, nous n'avons pu étudier qu'une petite partie de la variation dans la variable dépendante. C'est pourquoi nous voyons nos résultats comme un premier pas vers une analyse plus détaillée de la variation des taux de croissance des entreprises. Notre impression est que les facteurs très difficiles à cerner dans le cadre de sondages écrits, comme, par exemple, l'idée fondatrice, les structures formelles et informelles d'organisation ou bien la stratégie de l'entreprise, déterminent en grande partie le succès de l'entreprise. C'est ainsi que l'enquête de Kim et Mauborgne (1997), fondée sur une étude de cas, a montré que les entreprises les plus prospères sont justement celles qui remettent en question les pratiques de marché traditionnelles et qui poursuivent une «logique » stratégique autre qui ne tient pas compte des conditions de marché existantes. Les qualités psychologiques de l'entrepreneur, qui ne font pas partie de notre enquête, comme son ambition, son audace et son aspiration au profit, sont des facteurs explicatifs importants pour la croissance rapide des entreprises.

Nos résultats portent à croire qu'il y a, en effet, un petit nombre d'entreprises qui se distinguent par une forte croissance de l'emploi. Les entreprises à forte croissance se différencient par divers critères des entreprises «normales ». Si les responsables de la politique économique réussissent à identifier les entreprises à fort potentiel de croissance et à les encourager de façon ciblée, les effets sur l'emploi devraient être considérables. Le soutien économique peut se présenter sous la forme d'une amélioration des conditions générales institutionnelles, d'une amélioration du recrutement du personnel ou d'une aide au financement de l'expansion.

Revue internationale P.M.E., vol. 14, $\mathrm{n}^{\text {os }} 3-4,2001$ 


\section{Bibliographie}

Almus, M. (2000), What Characterizes a Fast Growing Firm?, Mannheim, ZEW, Cahier de recherche, $\mathrm{n}^{\circ}$ 00-64.

Almus, M. et E.A. NERLINGER (1999), « Growth of new technology-based firms - which factors matter? », Small Business Economics, vol. 13, n 2, p. 141-154.

Almus, M. et E.A. Nerlinger (2000), «Testing "Gibrat's Law" for young firms empirical results for West Germany », Small Business Economics, vol. 15, n 1 , p. 1-12.

AUDRETSCH, D. (1995), Innovation and Industry Evolution, Cambridge (MA), MIT Press.

BATES, T. (1990), «Entrepreneur human capital inputs and small business longevity », Review of Economics and Statistics, vol. 72, p. 551-559.

BECKER, G. (1993), Human Capital : A Theoretical and Empirical Analysis with Special Reference to Education, $3^{\mathrm{e}}$ édition, Chicago, The University of Chicago Press.

Beise, M., J. Felder, G. Licht, D. Harhoff, E. Nerlinger et H. Stahl (1995), Innovationsverhalten der Deutschen Wirtschaft - Innovationsaktivitäten kleiner und mittlerer Unternehmen, Bericht an das Bundesministerium für Bildung, Wissenschaft, Forschung und Technologie (BMBF), Mannheim, ZEW.

BIRCH, D. (1981), «Who creates jobs?», The Public Interest, vol. 65, no 1, p. 3-14.

BIRCH, D. (1987), Job Generation in America, New York, Free Press.

Boone, C., B. DE BrABANDER et A. VAN WitTELOOSTIJn (1996), « CEO locus of control and small firm performance : an integrative framework and empirical test », Journal of Management Studies, vol. 33, nº 4, p. 667-699.

Brock, W. et D. Evans (1986), The Economics of Small Businesses, New York, Holmes $\&$ Meier

BRÜDERL, J. et P. PREISENDÖRFER (2000), «Fast growing businesses, empirical evidence from a German study », International Journal of Sociology, vol. 30, n 1, p. 45-70.

BRÜDERL, J., P. PREISENDÖRfER et R. ZIEGLER (1992), «Survival chances of newly founded business organizations », American Sociological Review, vol. 57, $\mathrm{n}^{\circ} 2$, p. 227-241.

BRÜDERL, J., P. PREISENDÖRfER et R. ZIEGLER (1996), Der Erfolg neugegründeter Betriebe, New York, Holmes \& Meier.

CHILD, J. et A. KIESER (1983), Development of Organizations over Time, Handbook of Organizational Design, vol. 1: Adapting organizations to their environments, P.C. Nystrom et W.H. Starbuck (dir.), New York, Oxford University Press, p. 28-64.

DunNe, T., M. RoberTs et L. SAMUELSON (1989a), «Patterns of firm entry and exit in U.S. manufacturing industries », Rand Journal of Economics, vol. 19, n ${ }^{\circ} 4$, p. 495-515.

Dunne, T., M. Roberts et L. SAmuelson (1989b), «The growth and failure of U.S. manufacturing plants », Quarterly Journal of Economics, vol. 104, nº 4, p. 671-698.

EFER (1996), Europes's 500, Diegem.

Revue internationale P.M.E., vol. 14, $\mathrm{n}^{\text {os }} 3-4,2001$

(C) 2002 - Presses de l'Université du Québec

Édifice Le Delta I, 2875, boul. Laurier, bureau 450, Sainte-Foy, Québec G1V 2M2 • Tél. : (418) 657-4399 - www.puq.uquebec.ca

Tiré de : Revue internationale P.M.E., vol. 14, nos 3-4, sous la direction de Pierre-André Julien. 
Evans, D. (1987a), «The relationship between firm growth, size and age: estimates for 100 manufacturing industries », Journal of Industrial Economics, vol. 35, $\mathrm{n}^{\mathrm{o}} 4$, p. $567-581$.

Evans, D. (1987b), «Tests of alternative theories of firm growth », Journal of Political Economy, vol. 95, no 4, p. 657-674.

FinKElstein, S. et D. C. HAMBRICK (1990), « Top management team tenure and organizational outcomes : the moderating role of managerial discretion », Administrative Science Quarterly, vol. 35, no 4, p. 484-503.

FRANK, H. et C. KORUNKA (1996), «Zum informations- und entscheidungsverhalten von unternehmensgründern », Zeitschrift für Betriebswirtschaft, vol. 66, p. 947-963.

GiBrat, R. (1931), Les inégalités économiques, Paris, Librairie du recueil Sirey.

HALL, B. (1987), «The relationship between firm size and firm growth in the U.S. : manufacturing sector », Journal of Industrial Economics, vol. 35, nº 4, p. 583-606.

HARHOFF, D. et G. LiCHT (1994), «Das mannheimer Innovationspanel», dans H. Hochmuth et J. Wagner, Firmenpanelstudien in Deutschland, Tübingen, Basel, Mannheim, p. 255-284.

HARHOFF, D., K. STAHL et M. WoYWOdE (1998), «Legal form, growth and exit of West German firms : empirical results for manufacturing, construction, trade and service industries », Journal of Industrial Economics, vol. 46, n 4, p. 453-488.

Johnson, J., J. BALDWIN et C. Hinchley (1997), Successful Entrants : Creating the Capacity for Survival and Growth, Ottawa, Statistics Canada, Minister of Industry.

KANTER, R.M. (1994), When Giants Learn to Dance : Mastering the Challenges of Strategy, Management and Careers in the 190s, New York, Holmes \& Meier.

Katsoulacos, Y.S. (1986), The Employment Effect of Technical Change, Brighton, Wheatsheaf Books.

KIM, W.C. et R. MAUboRgne (1997), «Value innovation: the strategic logic of highgrowth », Harvard Business Review, janvier-février, p. 102-112.

Mendelson, H. (2000), «Organizational architecture and success in the information technology industry », Management Science, vol. 46, nº 4, p. 513-529.

Mendelson, H. et R. Pillai (1999), «Information age organizations, dynamics and performance », Journal of Economic Behavior \& Organization, vol. 38, n 2 , p. 253-281.

Milgrom, P. et J. Roberts (1992), Economics, Organization and Management, Englewood Cliffs, NJ, Prentice-Hall.

NonaKa, J. et H. TAKeuchi (1997), Die Organisation des Wissens : Wie japanische Unternehmen eine brachliegende Ressource nutzbar machen, Frankfurt a. M., Campus.

PENROSE, E. (1995), The Theory of the Growth of the Firm, zuerst veröffentlicht 1959, New York, Wiley.

PETERS, T. (1998), Der Innovationskreis : ohne Wandel kein Wachstum - wer abbaut verliert, München, Düsseldorf, Econ.

Revue internationale P.M.E., vol. 14, $\mathrm{n}^{\text {os }} 3-4,2001$ 
Peters, T.J. et R.H. WATERMAn (1994), Auf der Suche nach Spitzenleistungen: Was man von den bestgeführten US-Unternehmen lernen kann, $5^{\mathrm{e}}$ édition, München, MVG-Verlag.

PRAHAlAD, C.K. et G. HAMEL (1990), «The core competences of the corporation », Harvard Business Review, vol. 68, no 3, p. 79-91.

SEITZ, H. (1996), « Die Suburbanisierung der Beschäftigung : Eine empirische Analyse für Westdeutschland », Jahrbücher für Nationalökonomie und Statistik, 215, p. 69-98.

Simon, H (1996), Die heimlichen Gewinner - Hidden Champions, Frankfurt, Campus.

Simon, H.A. et C.P. Bonini (1956), «The size distribution of business firms », American Economic Review, vol. 48, p. 607-617.

StAhL, K. (1991), «Das Mannheimer Unternehmenspanel: Konzeption und Entwicklung », Mitteilungen aus der Arbeitsmarkt- und Berufsforschung, vol. 4, p. 735-738.

STEIL, F. et E. WOLF (1997), Welche Bedeutung haben Unternehmenscharakteristika und regionales Umfeld für die Beschäftigungsdynamik?, Cahier de recherche no 97-22, Mannheim, ZEW - Zentrum für Europäische Wirtschaftsforschung,

STOREY, D.J. (1995), Understanding the Small Business Sector, Londres, Routledge.

SutTon, J. (1997), «Gibrat's legacy », Journal of Economic Literature, vol. 35, nº 1, p. $40-59$.

WAGNER, J. (1994), «The post-entry performance of new small firms in German manufacturing industries », The Journal of Industrial Economics, vol. XLII, n 2 , p. 141-154.

Woywode, M. (1998), Determinanten der Überlebenswahrscheinlichkeit von Unternehmen, Baden-Baden, Nomos.

Revue internationale P.M.E., vol. 14, $\mathrm{n}^{\text {os }} 3-4,2001$ 